\title{
Sclerostin promotes human dental pulp cells senescence
}

\author{
Yanjing Ou ${ }^{1}$, Yi Zhou ${ }^{1}$, Shanshan Liang ${ }^{\text {Corresp.., }}{ }^{1}$, Yining Wang Corresp. 1 \\ ${ }^{1}$ The State Key Laboratory Breeding Base of Basic Science of Stomatology (Hubei-MOST) and Key Laboratory of Oral Biomedicine Ministry of Education, \\ School and Hospital of Stomatology, Wuhan University, Wuhan, China \\ Corresponding Authors: Shanshan Liang, Yining Wang \\ Email address: wb000867@whu.edu.cn, wang.yn@whu.edu.cn
}

Background. Senescence-related impairment of proliferation and differentiation limits the use of dental pulp cells for tissue regeneration. Deletion of sclerostin improves the dentinogenesis regeneration, while its role in dental pulp senescence is unclear. We investigated the role of sclerostin in subculture-induced senescence of human dental pulp cells (HDPCs) and in the senescence-related decline of proliferation and odontoblastic differentiation. Methods. Immunohistochemical staining and qRT-PCR analyses were performed to examine the expression pattern of sclerostin in young (20-to 30-year-old) and senescent (45- to 80-year-old) dental pulps. HDPCs were serially subcultured until senescence, and the expression of sclerostin was examined by qRT-PCR analysis. HDPCs with sclerostin overexpression and knockdown were constructed to investigate the role of sclerostin in HDPCs senescence and senescence-related impairment of odontoblastic differentiation potential. Results. By immunohistochemistry and qRT-PCR, we found a significantly increased expression level of sclerostin in senescent human dental pulp compared with that of young human dental pulp. Additionally, elevated sclerostin expression was found in subculture-induced senescent HDPCs in vitro. By sclerostin overexpression and knockdown, we found that sclerostin promoted HDPCs senescencerelated decline of proliferation and odontoblastic differentiation potential with increased expression of p16, p53 and p21 and downregulation of the Wnt signaling pathway. Discussion. The increased expression of sclerostin is responsible for the decline of proliferation and odontoblastic differentiation potential of HDPCs during cellular senescence. Anti-sclerostin treatment may be beneficial for the maintenance of the proliferation and odontoblastic differentiation potentials of HDPCs. 
1 Title: Sclerostin promotes human dental pulp cells senescence

2 Yanjing $\mathrm{Ou}^{1}$, Yi Zhou ${ }^{1}$, Shanshan Liang ${ }^{1}$, Yining Wang ${ }^{1}$

31 The State Key Laboratory Breeding Base of Basic Science of Stomatology (Hubei-MOST) and

4 Key Laboratory of Oral Biomedicine Ministry of Education, School and Hospital of Stomatology,

5 Wuhan University, Wuhan, China.

\section{Corresponding authors:}

7 Shanshan Liang and Yining Wang

8 E-mail: wb000867@whu.edu.cn and wang.yn@whu.edu.cn

9 


\section{Abstract}

11 Background. Senescence-related impairment of proliferation and differentiation limits the use of

12 dental pulp cells for tissue regeneration. Deletion of sclerostin improves the dentinogenesis

13 regeneration, while its role in dental pulp senescence is unclear. We investigated the role of

14 sclerostin in subculture-induced senescence of human dental pulp cells (HDPCs) and in the

15 senescence-related decline of proliferation and odontoblastic differentiation.

16 Methods. Immunohistochemical staining and qRT-PCR analyses were performed to examine the

17 expression pattern of sclerostin in young (20- to 30-year-old) and senescent (45- to 80-year-old)

18 dental pulps. HDPCs were serially subcultured until senescence, and the expression of sclerostin

19 was examined by qRT-PCR analysis. HDPCs with sclerostin overexpression and knockdown

20 were constructed to investigate the role of sclerostin in HDPCs senescence and senescence-

21 related impairment of odontoblastic differentiation potential.

22 Results. By immunohistochemistry and qRT-PCR, we found a significantly increased expression

23 level of sclerostin in senescent human dental pulp compared with that of young human dental

24 pulp. Additionally, elevated sclerostin expression was found in subculture-induced senescent

25 HDPCs in vitro. By sclerostin overexpression and knockdown, we found that sclerostin

26 promoted HDPCs senescence-related decline of proliferation and odontoblastic differentiation

27 potential with increased expression of $\mathrm{p} 16, \mathrm{p} 53$ and $\mathrm{p} 21$ and downregulation of the Wnt

28 signaling pathway.

29 Discussion. The increased expression of sclerostin is responsible for the decline of proliferation

30 and odontoblastic differentiation potential of HDPCs during cellular senescence. Anti-sclerostin 
31 treatment may be beneficial for the maintenance of the proliferation and odontoblastic

32 differentiation potentials of HDPCs.

\section{Introduction}

34 Dental caries, trauma, abrasion, attrition, erosion and dental treatment lead to tooth tissues

35 destruction, which eventually results in tooth loss. Non-biological treatment strategies for tooth

36 loss, such as bridges, dentures, and implants, may fit poorly or induce foreign body reaction and

37 run the risk of rejection by immune system (Modino \& Sharpe, 2005; Yen \& Sharpe, 2006).

Dental pulp cells (DPCs), a heterogeneous population of odontoblasts, epithelia, neurocytes, and

mesenchymal cells, possess self-renewal and pluripotent differentiation potentials and play a

crucial role in maintaining dental pulp homeostasis (Gronthos et al., 2002; Iohara et al., 2004).

Engineering tissue regeneration using DPCs as seed cells is a useful strategy to regenerate the

dentin-pulp complex, thereby lengthening the lifespan of teeth by preserving their defensive

ability (Gronthos et al., 2002; Karaoz et al., 2011; Potdar \& Jethmalani, 2015; Simon et al.,

44 2011).

45 In an aging society, fibrosis, atrophy, loss of cellularity, and degeneration of odontoblasts caused

by aging make it more difficult to maintain dental health of older individuals (Nakashima \&

47 Iohara, 2014). DPC senescence, caused by aging or other cytotoxic factors (e.g., oxidative stress

48 due to oral procedures (Soares et al., 2015) and irradation (Muthna et al., 2010)), is a state of

49 irreversible cellular arrest accompanied by aggregation of intracellular senescence molecules p16

50 and p53 (Rayess, Wang \& Srivatsan, 2012), and changes in secretion of bioactive soluble factors,

51 which lead to the decreased and then the arrest of proliferation and differentiation potentials 
52 (Iohara et al., 2014). However, little is known about the mechanisms underlying DPC senescence.

53 Previous studies demonstrated that several intracellular factors including p16, p21 and Bmi-1 are

54 involved in the progress of DPC senescence (Choi et al., 2012; Egbuniwe et al., 2011;

55 Mehrazarin et al., 2011). Recently, it was reported that the properties of senescent cells can be

56 reversed by changing the extrinsic microenvironment (Wagner et al., 2008; Nakashima \& Iohara,

57 2014). Therefore, identifying specific extrinsic factors involved in DPC senescence is of great

58 importance to biologically based tissue regeneration.

59 Sclerostin is a 190-amino acid secreted glycoprotein encoded by the SOST gene. Its expression is

60 restricted to the great arteries (Zhou et al., 2017), osteocytes (Compton \& Lee, 2014),

61 chondrocytes (Chan et al., 2011), and cementocytes (Bao et al., 2013). The deletion or down-

62 regulation of sclerostin causes high bone mass diseases such as sclerosteosis (van Lierop et al.,

63 2011) and Van Buchem disease (Loots et al., 2005). As a well-known negative regulator of bone

64 formation (Zhang et al., 2016), sclerostin inhibits the proliferation and differentiation of

65 cementoblasts (Bao et al., 2013) and osteoprogenitor cells including osteosarcoma cells (Zou,

66 Zhang \& Li, 2017) and human mesenchymal stem cells (Sutherland et al., 2004). Recently,

67 significantly elevated serum sclerostin was shown in the elderly (Modder et al., 2011; Roforth et

68 al., 2014), suggesting a possible role of sclerostin in aging-related bone loss as a result of

69 decreased regenerative potential caused by the accumulation of senescent cells (Farr et al.,

70 2017). In addition, previous studies demonstrated that the increased sclerostin produced by

71 osteoclasts from aged mice led to reduced bone formation (Ota et al., 2013), and anti-sclerostin

72 treatment increased bone mass in aged rats ( $\mathrm{Li}$ et al., 2009). Moreover, the activator of Sirt1, a 
73 critical regulator of aging and longevity (Satoh et al., 2013), rescued ovariectomy-induced bone

74 loss by decreasing sclerostin expression (Artsi et al., 2014). Therefore, it was speculated that

75 sclerostin might impact cellular senescence in terms of differentiation and proliferation.

76 Similar to the higher level of sclerostin in aged individuals, the expression level of sclerostin

77 varies in embryonic and adult mouse incisors and molars (Naka \& Yokose, 2011), indicating a

78 possible role of sclerostin in DPC senescence. Additionally, Collignon et al. found that sclerostin

79 deficiency increased reparative dentinogenesis in mice (Collignon et al., 2017), implying anti-

80 sclerostin might reverse the deccreased regenerative potential of aged dental pulp. Taken

81 together, we hypothesized a possible correlation between sclerostin and DPC senescence.

82 Therefore, the purpose of our study was to elucidate the role of sclerostin in the process of

83 human dental pulp cell (HDPC) senescence as well as aging-related impairment of HDPC

84 proliferation and odontoblastic differentiation.

85 Materials and Methods

86 This work was carried out in accordance with the World Medical Association Declaration of

87 Helsinki (World Medical, 2013). The protocols and procedures were reviewed and approved by

88 the Ethical Committee of the School and Hospital of Stomatology, Wuhan University, China

89 (2015C12).

\section{Human dental pulp collection}

91 Healthy and fresh human premolars were extracted from 20- to 80-year-old patients who were

92 under orthodontic or periodontal treatment in the Hospital of Stomatology, Wuhan University.

93 All donors gave their informed consent. The teeth were divided into two groups: the young group 
94 contained 30 teeth from 20 - to 30 -year-old patients and the old group contained 20 teeth from

95 45- to 80-year-old patients.

96 Ten teeth from each group were used for immunohistochemical analysis, and ten teeth from each

97 group for qRT-PCR analysis. The remaining 10 teeth from the young group were used for HDPC

98 culture.

\section{Immunohistochemical analysis}

100 Teeth were fixed with $4 \%$ paraformaldehyde at room temperature and decalcified in $10 \%$ EDTA

101 solution for more than 6 months. Teeth were cut into 5- $\mu$ m-thick serial sections, which were

102 collected on poly-L-lysine-coated slides. For immunohistochemical staining, the sections were

103 dewaxed and incubated with rabbit anti-sclerostin (1:200; Abclonal, Boston, USA) at $4^{\circ} \mathrm{C}$

104 overnight. The staining was performed using a biotin-streptavidin kit (ZhongShan Biotech,

105 Beijing, China) before the sections were counterstained with hematoxylin.

\section{Real-time PCR analysis}

107 Total RNA was isolated using the RNAiso kit (Takara, Japan). First-strand cDNA syntheses

108 were performed by using the PrimeScript ${ }^{\mathrm{TM}}$ RT reagent Kit with gDNA Eraser (Takara). Real-

109 time polymerase chain reaction for sclerostin, p16, p53, p21, alkaline phosphatase (ALP),

110 osteocalcin (OCN), osteocalcin (OPN), dentin sialophosphoprotein (DSPP) and glyceraldehyde-

111 3-phosphate-dehydrogenase (GAPDH) was performed with the SYBR Green Kit (Takara) using

112 a Applied BioSystems 7900HT thermocycler (Applied Biosystems, CA. USA). The primers are

113 listed in Table 1. The relative amount or fold change of the target gene was normalized relative

114 to the level of human GAPDH and the control groups. 


\section{Cells and cell culture}

116 HDPCs were isolated from the healthy dental pulp of 10 premolars from young group. The

117 dental pulp tissues were digested in a solution containing $4 \mathrm{mg} / \mathrm{mL}$ dispase and $3 \mathrm{mg} / \mathrm{mL}$

118 collagenase type I for 1 hour at $37^{\circ} \mathrm{C}$ and then cultured in $\alpha$-modified essential medium $(\alpha-\mathrm{MEM}$;

119 HyClone Laboratories, Inc, UT, USA) containing 10\% fetal bovine serum (FBS; Gibco, NY, $120 \mathrm{USA}$ ) at $37^{\circ} \mathrm{C}$ under $5 \% \mathrm{CO}_{2}$. Confluent monolayers were dissociated with $0.5 \%(\mathrm{w} / \mathrm{v})$ trypsin-

121 EDTA for subculture. HDPCs were serially subcultured until the cells spontaneously arrested 122 their replication. In brief, $2 \times 10^{5} \mathrm{HDPCs}$ were plated in $60 \mathrm{~mm}$ diameter dishes and the medium 123 was changed every 3 days. When the cells reach $80 \%$ confluence, all cells were dissociated with $1240.5 \%(\mathrm{w} / \mathrm{v})$ trypsin-EDTA and counted by a cell counting device (Vi-CELL ${ }^{\mathrm{TM}} \mathrm{XR}$, Beckman 125 Coulter, CA, USA). The number of population doublings (PD) was calculated using the 126 following formula: $\mathrm{PD}=\log _{2}$ (cells harvested/cells seeded) + previous PD (Huh et al., 2016;

127 Kitajima et al., 2011). For odontoblastic induction, the medium was changed to the odontoblastic 128 induction medium, containing $\alpha$-MEM, 5\% FBS, $100 \mathrm{U} / \mathrm{mL}$ penicillin, $100 \mu \mathrm{g} / \mathrm{mL}$ streptomycin, $12910 \mathrm{mmol} / \mathrm{L} \beta$-glycerophosphate, $50 \mu \mathrm{g} / \mathrm{mL}$ ascorbic acid, and $10 \mathrm{nmol} / \mathrm{L}$ dexamethasone (Sigma130 Aldrich Co, MO, USA). Cultures were maintained with a medium change every 3 days.

\section{Lentivirus packaging and cell infection}

132 Full human SOST cds sequence and a human SOST sh-RNA were cloned and inserted into the 133 PCDH-CMV-MCS-EF1-copGFP vector (GenePharma, China) and the GV298 vector

134 (Genechem, China), respectively. Lentiviral particles were produced using three-plasmid systems,

135 including pMD2.G, psPAX2 and the individual vectors, with NEOFECT ${ }^{\mathrm{TM}}$ DNA transfection 136 reagent (Neofect, China) according to the manufacturer's instruction. For infection, HDPCs were 
137 incubated with lentiviral particles and polybrene $(4 \mu \mathrm{g} / \mathrm{mL})$ in complete medium for 12 hours.

138 Cells with successful infection by pCDH-human-SOST were designated SOST, cells infected by

139 sh-SOST were designated sh-SOST, and control cells infected by empty vector were designated

140 Control and sh-Ctrl, respectively. The expression of sclerostin was quantified by real-time PCR

141 and Western blot analysis. The mRNA and protein expression levels were significantly

142 upregulated in the SOST group $(P<0.001$, Fig. 1E and F), while they were knocked down by

143 over $85 \%$ in the sh-SOST group 48 hours after infection $(P<.0001$, Fig. $1 \mathrm{G}$ and $\mathrm{H})$.

144 Western blot

145 Cells were lysed in M-PER Mammalian Protein Extraction Reagent (Thermo, IL, USA)

146 combined with a cocktail of protease inhibitors (Roche Molecular Biochemicals, Rotkreuz,

147 Switzerland). Total lysate was loaded and separated by SDS/PAGE. Primary antibodies specific

148 for sclerostin (1:1000, Abclonal), phosphor- $\beta$-catenin (p- $\beta$-catenin) $(1: 1000$, Cell Signaling

149 Technology, MA, USA), and $\beta$-actin (1:5000, Santa Cruz, CA, USA) were used.

150 Senescence-associated $\boldsymbol{\beta}$-galactosidase assay (SA- $\boldsymbol{\beta}$-Gal)

151 To determine senescence of HDPCs, an SA- $\beta$-Gal kit (Beyotime, China) was used according to

152 the manufacturer's instructions. In brief, cells seeded on slips were fixed with paraformaldehyde

153 and incubated with SA- $\beta-$ Gal overnight. Senescent HDPCs were identified by blue-staining

154 under standard light microscopy.

155 Cell proliferation assay

156 HDPCs were seeded at a density of $2 \times 10^{3}$ cells/well in a 96-well plate and cultured for 24 hours,

157 and then the medium was replaced with fresh medium. On days 1, 2, 3, and 4, the density of 
158 viable cells within each well was quantified with the Cell Counting Kit-8 (CCK-8; Dojindo,

159 Japan) according to the manufacturer's protocols. The absorbance at $450 \mathrm{~nm}$ was measured to

160 calculate the number of viable cells in each well. A well with medium and CCK-8 solution but

161 without cells was used as the baseline.

\section{Alkaline phosphatase staining and ALP activity assay}

163 ALP staining was performed according to the manufacturer's instructions (Beyotime). For ALP

164 activity, cells were lysed with $0.1 \%$ Triton $\mathrm{X}-100$, and $50 \mu \mathrm{L}$ lysate was mixed with $100 \mu \mathrm{L}$ p-

165 nitrophenyl phosphate $(4 \mathrm{mg} / \mathrm{mL})$. The mixture was incubated at $37^{\circ} \mathrm{C}$ for $15-20$ minutes. The

166 reaction was stopped by the addition of $0.5 \mathrm{~N} \mathrm{NaOH}(100 \mu \mathrm{L})$ and read spectrophotometrically at

$167405 \mathrm{~nm}$. The protein concentration of the lysate was determined as described in the

168 manufacturer's instructions of the Pierce BCA protein assay kit (Thermo). The enzyme activity

169 was quantified by a p-nitrophenol standard curve and normalized by protein concentration.

\section{Alizarin red staining}

171 Cells were cultured in 12-well cell culture dishes in the odontoblastic induction medium for 14

172 days. Then, cells were fixed with $4 \%$ paraformaldehyde and stained with $2 \%$ alizarin red. The

173 stain was desorbed with $10 \%$ cetylpyridinium chloride for 1 hour and the absorbance was

174 examined at $562 \mathrm{~nm}$.

\section{Statistical analysis}

176 All experiments were repeated at least three times. Quantitative results are expressed as the mean

$177 \pm$ standard deviation. Data were analyzed by t-test and one-way analyses of variance using SPSS

17819.0 software (SPSS Inc, IL, USA). $P$ values $<0.05$ were considered statistically significant. 


\section{Results}

180 Expression of sclerostin is increased in senescent human dental pulp and subculture-

\section{1 induced senescent HDPCs}

182 The immunohistochemistry assay showed that the expression of sclerostin in young human pulp was very low, and positive sclerostin staining was only found in odontoblasts of young dental pulp. In contrast, strong staining of sclerostin was observed throughout the senescent human qRT-PCR analyses showed a higher expression level of sclerostin mRNA in senescent dental pulp, along with increased expression levels of p16, p53, and p21 (Figure 1C).

Rapidly proliferating HDPCs were serially subcultured until the cells spontaneously arrested replication. HDPCs completing 16 and 54 population doublings (PDs) were defined as young and senescent HDPCs, respectively (Mehrazarin et al., 2011). In line with the higher expression levels of sclerostin, p16, p53, and p21 in senescent dental pulp, qRT-PCR analyses showed significantly higher expression levels of sclerostin, p16, p53, and p21 in senescent HDPCs

193 (Figure 1D).

\section{Sclerostin induces HDPCs senescence}

195 To access the role of sclerostin in senescence of HDPCs, sclerostin was overexpressed in early-

196 passaged HDPCs. As shown in Figure 2, sclerostin overexpression significantly increased the

197 number of SA- $\beta$-Gal-positive cells. In addition, the CCK-8 assay showed significantly decreased

198 proliferation and higher expression levels of p16, p53, and p21 in sclerostin-overexpressing

199 HDPCs (Figure 2C and D). In contrast, knockdown of sclerostin in late-passaged HDPCs 
200 showed fewer SA- $\beta$-Gal-positive cells, increased proliferation, and decreased p16, p53 and p21

201 expressions (Figure 2E-H). In conclusion, these results suggest that sclerostin induces HDPCs

202 senescence and inhibits HDPCs proliferation via the p16 and p53 signaling pathways.

\section{Sclerostin inhibits odontoblastic differentiation of HDPCs}

204 To determine the effect of sclerostin on HDPC differentiation, we compared the odontoblastic

205 differentiation ability between early-passaged SOST and Control groups. As shown in Figure 3,

206 sclerostin overexpression significantly decreased the odontoblastic differentiation of early-

207 passaged HDPCs as shown by the decreased mineralization nodules formation and a decline in

208 ALP activity along with lower expression levels of odontoblastic differentiation markers such as

209 ALP, OPN, CON, and DSPP (Figure 3A-C). Moreover, knockdown of sclerostin rescued the

210 decreased odontoblastic differentiation of senescent HDPCs (Figure 3D-E). These data imply

211 that the increased expression of sclerostin contributed to the impaired odontoblastic

212 differentiation potential of senescent HDPCs.

213 The Wnt/ $\beta$-catenin pathway may be involved in the progress of HDPCs senescence related

\section{4 to higher expression of sclerostin}

215 To clarify the mechanisms underlying sclerostin-related HDPCs senescence, we examined the

$216 \mathrm{Wnt} / \beta$-catenin pathway activity. Western blot analysis showed that overexpression of sclerostin

217 suppressed the activity of the $\mathrm{Wnt} / \beta$-catenin pathway by increasing the expression of $\mathrm{p}$ - $\beta$-catenin

218 and sclerostin knockdown significantly increased the activity of the $\mathrm{Wnt} / \beta$-catenin pathway

219 (Figure 4). These results suggest that sclerostin might accelerate senescence of HDPCs in a Wnt

220 signaling pathway-dependent mechanism. 


\section{Discussion}

222 The importance of dental pulp cells in diverse therapeutic application has been increasingly

223 recognized in recent years. However, the impairment of proliferation and differentiation caused

224 by cellular senescence restricts their application in tissue regeneration. So far, little is known

225 about the progress of HDPC senescence. Herein, by sclerostin overexpression in early-passaged

226 HDPCs and knockdown in late-passaged HDPCs, we showed that sclerostin knockdown is

227 beneficial for the maintenance of the proliferation and odontoblastic differentiation potentials of

228 HDPCs during cellular senescence.

229 In the present study, we found that there was a significantly higher expression of sclerostin in

230 senescent human dental pulp tissues and senescent HDPCs. These data are consistent with the

231 findings that serum sclerostin levels increase with age, which may contribute to age related bone

232 loss (Modder et al., 2011; Zhang et al., 2016). While noteworthy, these results do not provide

233 causality. Therefore, SA- $\beta$-Gal staining, a biomarker of cellular senescence (Debacq-Chainiaux

234 et al., 2009), was performed in early-passaged HDPCs with sclerostin overexpression and in late-

235 passaged HDPCs with sclerostin knockdown to determine whether the higher expression of

236 sclerostin was the cause of HDPC senescence or not. Sclerostin overexpression accelerated

237 HDPC senescence and sclerostin knockdown decreased senescence of HDPCs in vitro. To the

238 best of our knowledge, it was the first study confirming the exact role of sclerostin in cellular

239 senescence. Well-designed studies are now required to determine the role of sclerostin in HDPC

240 senescence in vivo and to identify whether this promoting effect of sclerostin on HDPC

241 senescence is universal in other cells or is cell-type specific.

242 Senescence of cells is mostly due to activation of G1/S cell cycle arrest proteins (Itahana,

243 Campisi \& Dimri, 2004). The p53/p21 and p16/retinoblastoma axes are two important pathways 
244 in cellular senescence. The p16 protein mediates cell cycle arrest by inhibiting DNA replication

245 via preventing phosphorylation of the retinoblastoma protein (Rayess, Wang \& Srivatsan, 2012).

246 The p53-mediated response to DNA damage, oxidants and hypoxia induces the expression of

247 p21, leading to cellular senescence by inhibiting the activity of cyclin dependent kinases

248 (Muthna et al., 2010; Tonnessen-Murray, Lozano \& Jackson, 2017). Our study showed that

249 senescent human dental pulp and subculture-induced senescent HDPCs exhibited higher 250 expression levels of $\mathrm{p} 16, \mathrm{p} 53$ and $\mathrm{p} 21$. These data are in accordance with previous findings that

251 p16, p53 and p21 are highly expressed in senescent dental pulp cells (Mas-Bargues et al., 2017;

252 Muthna et al., 2010). Conversely, downregulation of p53-p21 decreased senescence of dental

253 pulp stem cells (Choi et al., 2012). Meanwhile, p16 knockdown significantly reduced

254 senescence-related dysfunction of dental pulp mesenchymal stem cells (Feng et al., 2014).

255 Inspired by the similar expression profile of p16, p53, p21 and sclerostin during HDPCs

256 senescence, we examined the effect of sclerostin on the expression of p16, p53 and p21 in

257 HDPCs. Sclerostin overexpression significantly increased the expression levels of p16, p53 and

258 p21, whereas, sclerostin knockdown decreased the expression levels of p16, p53 and p21.

259 Although, there had previously been no reports about the effect of sclerostin on the expression of

$260 \mathrm{p} 16, \mathrm{p} 53$ and $\mathrm{p} 21$, it was believed that senescence acted as a tumor suppressor and sclerostin

261 silence significantly increased the proliferation of osteosarcoma cells by promoting the progress

262 of cell cycle in G1/S phase (Zou, Zhang \& Li, 2017). Thus, one can conclude that sclerostin may

263 modulate the progression of HDPC senescence via both the p16 and p53 pathways.

264 Odontoblastic differentiation potential was impaired in senescent HDPCs with decreased

265 expression levels of odontoblastic differentiation markers ALP, OCN, OPN and DSPP, which

266 play key roles in matrix formation and calcification initialization in bone and teeth (Bae et al., 
267 2015; Chen et al., 2005; Kuratate et al., 2008; Ma et al., 2009; Min et al., 2010). In this study, we

268 found that sclerostin significantly inhibited odontoblastic differentiation of early-passaged

269 HDPCs with downregulation of odontoblastic markers ALP, OCN, OPN, and DSPP.

270 Furthermore, knockdown of sclerostin increased odontoblastic differentiation of subculture-

271 induced senescent HDPCs with higher expression levels of these odontoblastic differentiation

272 markers. These results are in line with the finding that mice dental pulp cells with sclerostin

273 deficiency exhibited enhanced in vitro mineralization (Mehrazarin et al., 2011). These results

274 indicate that the increased expression of sclerostin was responsible for impairment of

275 odontoblastic differentiation potential in senescent HDPCs.

276 It was reported that sclerostin efficiently inhibited Wnt signaling by interrupting the Wnt-

277 Frizzled-LRP5/6 receptor complex via binding with the Wnt co-receptor LRP5/6 (Semenov,

278 Tamai \& $\mathrm{Xi}, 2005)$, leading to upregulated expression of $\mathrm{p}-\beta$-catenin, thereby preventing the

279 nucleus translocation of stabilized $\beta$-catenin and thus downregulating downstream genes

280 expression (Bae et al., 2015). Wnt signaling plays an essential role in age-related changes in stem

281 cells. Previous studies found that inhibiting Wnt signaling initiated senescence of glioblastoma

282 cells and human WI38 fibroblasts (Lambiv et al., 2011; Ye et al., 2007). Furthermore, Wnt1, an

283 agonist of Wnt signaling, rescued the impaired neurogenic differentiation potential of aged

284 dental pulp stem cells (Feng et al., 2013). In this study, sclerostin overexpression attenuated the 285 activity of Wnt signaling and sclerostin knockdown activated Wnt signaling. These results 286 indicate that sclerostin might accelerate senescence of HDPCs, in part, by decreasing Wnt 287 signaling. However, the exact mechanism of sclerostin-related HDPC senescence requires further 288 study to elucidate.

289 Conclusion 
290 Taken together, the higher expression of sclerostin might accelerate HDPC senescence and was

291 responsible for the attenuated proliferative and odontoblastic differentiation potentials in

292 senescent HDPCs via p16 and p53 pathways. Sclerostin may serve as a target to delay the

293 progress of HDPCs senescence.

294 Acknowledgements

295 We thank M.D.S. Chufang Liao (School and Hospital of Stomatology, Wuhan University) and 296 M.D.S. Yun Wu (School and Hospital of Stomatology, Wuhan University) for HDPC 297 preparation and for their advice on our work.

\section{References}

299 Artsi H, Cohen-Kfir E, Gurt I, Shahar R, Bajayo A, Kalish N, Bellido TM, Gabet Y, Dresner-

300 Pollak R. 2014. The Sirtuin1 activator SRT3025 down-regulates sclerostin and rescues

301 ovariectomy-induced bone loss and biomechanical deterioration in female mice. Endocrinology

302 155:3508-3515 DOI: 10.1210/en.2014-1334.

303 Bae CH, Kim TH, Ko SO, Lee JC, Yang X, Cho ES. 2015. Wntless regulates dentin apposition

304 and root elongation in the mandibular molar. Journal of Dental Research 94:439-445 DOI:

$30510.1177 / 0022034514567198$.

306 Bao XF, Liu YY, Han GH, Zuo ZG, Hu M. 2013. The effect on proliferation and differentiation

307 of cementoblast by using sclerostin as inhibitor. International Journal of Molecular Sciences

308 14:21140-21152 DOI:10.3390/ijms 141021140.

309 Chan BY, Fuller ES, Russell AK, Smith SM, Smith MM, Jackson MT, Cake MA, Read RA,

310 Bateman JF, Sambrook PN, Little CB. 2011. Increased chondrocyte sclerostin may protect

311 against cartilage degradation in osteoarthritis. Osteoarthritis Cartilage 19:874-885 DOI:

312 10.1016/j.joca.2011.04.014. 
313 Chen S, Rani S, Wu YM, Unterbrink A, Gu TT, Gluhak-Heinrich J, Chuang HH, MacDougall M.

314 2005. Differential regulation of dentin sialophosphoprotein expression by Runx2 during 315 odontoblast cytodifferentiation. Journal of Biological Chemistry 280:29717-29727 DOI: 316 10.1074/jbc.M502929200.

317 Choi YJ, Lee JY, Chung CP, Park YJ. 2012. Cell-penetrating superoxide dismutase attenuates 318 oxidative stress-induced senescence by regulating the p53-p21(Cip1) pathway and restores 319 osteoblastic differentiation in human dental pulp stem cells. International Journal of 320 Nanomedicine 7:5091-5106 DOI: 10.2147/IJN.S31723.

321 Collignon AM, Amri N, Lesieur J, Sadoine J, Ribes S, Menashi S, Simon S, Berdal A, Rochefort

322 GY, Chaussain C, Gaucher C. 2017. Sclerostin deficiency promotes reparative dentinogenesis.

323 Journal of Dental Research 96:815-821 DOI: 10.1177/0022034517698104.

324 Compton JT, Lee FY. 2014. A review of osteocyte function and the emerging importance of 325 sclerostin. Journal of Bone and Joint Surgery 96:1659-1668 DOI: 10.2106/JBJS.M.01096.

326 Debacq-Chainiaux F, Erusalimsky JD, Campisi J, Toussaint O. 2009. Protocols to detect 327 senescence-associated beta-galactosidase (SA-betagal) activity, a biomarker of senescent cells in 328 culture and in vivo. Nature Protocols 4:1798-1806 DOI: 10.1038/nprot.2009.191.

329 Farr JN, Xu M, Weivoda MM, Monroe DG, Fraser DG, Onken JL, Negley BA, Sfeir JG, 330 Ogrodnik MB, Hachfeld CM, LeBrasseur NK, Drake MT, Pignolo RJ, Pirtskhalava T, Tchkonia 331 T, Oursler MJ, Kirkland JL, Khosla S. 2017. Targeting cellular senescence prevents age-related 332 bone loss in mice. Nature Medicine 23:1072-1079 DOI: 10.1038/nm.4385.

333 Feng XM, Xing J, Feng GJ, Huang D, Lu XH, Liu SZ, Tan W, Li LR, Gu ZF. 2014. p16(INK4A) 334 mediates age-related changes in mesenchymal stem cells derived from human dental pulp 
335 through the DNA damage and stress response. Mechanisms of Ageing and Development 141:46-

33655 DOI: 10.1016/j.mad.2014.09.004.

337 Feng XM, Xing J, Feng GJ, Sang AM, Shen BY, Xu Y, Jiang JX, Liu SZ, Tan W, Gu ZF, Li LR.

338 2013. Age-dependent impaired neurogenic differentiation capacity of dental stem cell is

339 associated with Wnt/ $\beta$-catenin signaling. Cellular and Molecular Neurobiology 33:1023-1031

340 DOI: 10.1007/s10571-013-9965-0.

341 Gronthos S, Brahim J, Li W, Fisher LW, Cherman N, Boyde A, DenBesten P, Robey PG, Shi S.

342 2002. Stem cell properties of human dental pulp stem cells. Journal of Dental Research 81:531-

343535 DOI: 10.1177/154405910208100806.

344 Huh CJ, Zhang B, Victor MB, Dahiya S, Batista LF, Horvath S, Yoo AS. 2016. Maintenance of

345 age in human neurons generated by microRNA-based neuronal conversion of fibroblasts. Elife 5:

346 e18648 DOI: $10.7554 /$ eLife. 18648.

347 Iohara K, Nakashima M, Ito M, Ishikawa M, Nakasima A, Akamine A. 2004. Dentin 348 regeneration by dental pulp stem cell therapy with recombinant human bone morphogenetic 349 protein 2. Journal of Dental Research 83:590-595 DOI: 10.1177/154405910408300802.

350 Iohara K, Murakami M, Nakata K, Nakashima M. 2014. Age-dependent decline in dental pulp 351 regeneration after pulpectomy in dogs. Experimental Gerontology 52: 39-45 DOI: 352 10.1016/j.exger.2014.01.020.

353 Itahana K, Campisi J, Dimri GP. 2004. Mechanisms of cellular senescence in human and mouse 354 cells. Biogerontology 5:1-10 DOI: 10.1023/B:BGEN.0000017682.96395.10.

355 Karaoz E, Demircan PC, Saglam O, Aksoy A, Kaymaz F, Duruksu G. 2011. Human dental pulp 356 stem cells demonstrate better neural and epithelial stem cell properties than bone marrow-derived 
357 mesenchymal stem cells. Histochemistry and Cell Biology 136:455-473 DOI: 10.1007/s00418$358 \quad 011-0858-3$.

359 Kitajima S, Miki T, Takegami Y, Kido Y, Noda M, Hara E, Shamma A, Takahashi C. 2011.

360 Reversion-inducing cysteine-rich protein with Kazal motifs interferes with epidermal growth

361 factor receptor signaling. Oncogene 30:737-750 DOI: 10.1038/onc.2010.448.

362 Kuratate M, Yosbiba K, Sbigetani Y, Yosbiba N, Obsbima H, Okiji T. 2008.

363 Immunohistochemical analysis of nestin, osteopontin, and proliferating cells in the reparative

364 process of exposed dental pulp capped with mineral trioxide aggregate. Journal of Endodontics 365 34:970-974 DOI: 10.1016/j.joen.2008.03.021.

366 Lambiv WL, Vassallo I, Delorenzi M, Shay T, Diserens AC, Misra A, Feuerstein B, Murat A,

367 Migliavacca E, Hamou MF, Sciuscio D, Burger R, Domany E, Stupp R, Hegi ME. 2011. The

368 Wnt inhibitory factor 1 (WIF1) is targeted in glioblastoma and has a tumor suppressing function

369 potentially by induction of senescence. Neuro-Oncology 13:736-747 DOI:

$370 \quad 10.1093 /$ neuonc/nor036.

371 Li XD, Ominsky MS, Warmington KS, Morony S, Gong JH, Cao J, Gao YM, Shalhoub V,

372 Tipton B, Haldankar RJ, Chen Q, Winters A, Boone T, Geng ZP, Niu QT, Ke HZ, Kostenuik PJ,

373 Simonet WS, Lacey DL, Paszty C. 2009. Sclerostin antibody treatment increases bone formation,

374 bone mass, and bone strength in a rat model of postmenopausal osteoporosis. Journal of Bone 375 and Mineral Research 24:578-588 DOI: 10.1359/jbmr.081206.

376 Loots GG, Kneissel M, Keller H, Baptist M, Chang J, Collette NM, Ovcharenko D, Plajzer-Frick

377 I, Rubin EM. 2005. Genomic deletion of a long-range bone enhancer misregulates sclerostin in

378 Van Buchem disease. Genome Research 15:928-935 DOI: 10.1101/gr.3437105. 
379 Ma DD, Ma ZF, Zhang XJ, Wang WH, Yang ZH, Zhang M, Wu G, Lu W, Deng ZH, Jin Y.

380 2009. Effect of age and extrinsic microenvironment on the proliferation and osteogenic

381 differentiation of rat dental pulp stem cells in vitro. Journal of Endodontics 35:1546-1553 DOI:

382 10.1016/j.joen.2009.07.016.

383 Mas-Bargues C, Vina-Almunia J, Ingles M, Sanz-Ros J, Gambini J, Ibanez-Cabellos JS, Garcia-

384 Gimenez JL, Vina J, Borras C. 2017. Role of p16(INK4a) and BMI-1 in oxidative stress-induced 385 premature senescence in human dental pulp stem cells. Redox Biology 12:690-698 DOI: 386 10.1016/j.redox.2017.04.002.

387 Mehrazarin S, Oh JF, Chung CL, Chen W, Kim RH, Shi ST, Park NH, King MK. 2011. Impaired 388 odontogenic differentiation of senescent dental mesenchymal stem cells is associated with loss of 389 bmi-1 expression. Journal of Endodontics 37:662-666 DOI: 10.1016/j.joen.2011.02.009.

390 Min KS, Lee YM, Hong SO, Kim EC. 2010. Simvastatin promotes odontoblastic differentiation 391 and expression of angiogenic factors via heme oxygenase-1 in primary cultured human dental 392 pulp cells. Journal of Endodontics 36:447-452 DOI: 10.1016/j.joen.2009.11.021.

393 Modder UI, Hoey KA, Amin S, McCready LK, Achenbach SJ, Riggs BL, Melton LJ, Khosla S. 394 2011. Relation of age, gender, and bone mass to circulating sclerostin levels in women and men. 395 Journal of Bone and Mineral Research 26:373-379 DOI: 10.1002/jbmr.217.

396 Modino SAC, Sharpe PT. 2005. Tissue engineering of teeth using adult stem cells. Archives of 397 Oral Biology 50:255-258 DOI: 10.1016/j.archoralbio.2005.01.002.

398 Muthna D, Soukup T, Vavrova J, Mokry J, Cmielova J, Visek B, Jiroutova A, Havelek R, 399 Suchanek J, Filip S, English D, Rezacova M. 2010. Irradiation of adult human dental pulp stem 400 cells provokes activation of p53, cell cycle arrest, and senescence but not apoptosis. Stem Cells 401 and Development 19:1855-1862 DOI: 10.1089/scd.2009.0449. 
402 Naka T, Yokose S. 2011. Spatiotemporal expression of sclerostin in odontoblasts during 403 embryonic mouse tooth morphogenesis. Journal of Endodontics 37:340-345 DOI: 404 10.1016/j.joen.2010.11.025.

405 Nakashima M, Iohara K. 2014. Mobilized dental pulp stem cells for pulp regeneration: initiation 406 of clinical trial. Journal of Endodontics 40:S26-32 DOI: 10.1016/j.joen.2014.01.020.

407 Ota K, Quint P, Ruan M, Pederson L, Westendorf JJ, Khosla S, Oursler MJ. 2013. Sclerostin is 408 expressed in osteoclasts from aged mice and reduces osteoclast-mediated stimulation of 409 mineralization. Journal of Cellular Biochemistry 114:1901-1907 DOI: 10.1002/jcb.24537.

410 Potdar PD, Jethmalani YD. 2015. Human dental pulp stem cells: Applications in future 411 regenerative medicine. World Journal of Stem Cells 7:839-851 DOI: 10.4252/wjsc.v7.i5.839.

412 Rayess H, Wang MB, Srivatsan ES. 2012. Cellular senescence and tumor suppressor gene p16. 413 International Journal of Cancer 130:1715-1725 DOI: 10.1002/ijc.27316.

414 Roforth MM, Fujita K, McGregor UI, Kirmani S, McCready LK, Peterson JM, Drake MT, 415 Monroe DG, Khosla S. 2014. Effects of age on bone mRNA levels of sclerostin and other genes 416 relevant to bone metabolism in humans. Bone 59:1-6 DOI: 10.1016/j.bone.2013.10.019.

417 Satoh A, Brace CS, Rensing N, Cliften P, Wozniak DF, Herzog ED, Yamada KA, Imai S. 2013. 418 Sirt1 extends life span and delays aging in mice through the regulation of Nk2 homeobox 1 in 419 the DMH and LH. Cell Metabolism 18:416-430 DOI: 10.1016/j.cmet.2013.07.013.

420 Semenov M, Tamai K, Xi H. 2005. SOST is a ligand for LRP5/LRP6 and a Wnt signaling 421 inhibitor. Journal of Biological Chemistry 280:26770-26775 DOI: 10.1074/jbc.M504308200.

422 Simon SR, Berdal A, Cooper PR, Lumley PJ, Tomson PL, Smith AJ. 2011. Dentin-pulp complex 423 regeneration: from lab to clinic. Advances in Dental Research 23:340-345 DOI: $42410.1155 / 2012 / 190561$. 
425 Soares DG, Basso FG, Hebling J, Costa CAD. 2015. Effect of hydrogen-peroxide-mediated 426 oxidative stress on human dental pulp cells. Journal of Dentistry 43:750-756 DOI: 427 10.1016/j.jdent.2014.12.006.

428 Sutherland MK, Geoghegan JC, Yu CP, Turcott E, Skonier JE, Winkler DG, Latham JA. 2004. 429 Sclerostin promotes the apoptosis of human osteoblastic cells: a novel regulation of bone 430 formation. Bone 35:828-835 DOI: 10.1016/j.bone.2004.05.023.

431 Tonnessen-Murray CA, Lozano G, Jackson JG. 2017. The regulation of cellular functions by the 432 p53 protein: cellular senescence. Cold Spring Harbor Perspectives in Medicine 7 DOI: 433 10.1101/cshperspect.a026112.

434 van Lierop AH, Hamdy NAT, Hamersma H, van Bezooijen RL, Power J, Loveridge N, 435 Papapoulos SE. 2011. Patients with sclerosteosis and disease carriers: human models of the 436 effect of sclerostin on bone turnover. Journal of Bone and Mineral Research 26:2804-2811 DOI: $43710.1002 / \mathrm{jbmr} .474$.

438 Wagner W, Horn P, Bork S, Ho AD. 2008. Aging of hematopoietic stem cells is regulated by the 439 stem cell niche. Experimental Gerontology 43:974-80 DOI: 10.1016/j.exger.2008.04.007.

440 World Medical A. 2013. World Medical Association Declaration of Helsinki: ethical principles 441 for medical research involving human subjects. JAMA-Journal of the American Medical 442 Association 310:2191-2194 DOI: 10.1001/jama.2013.281053.

443 Ye XF, Zerlanko B, Kennedy A, Banumathy G, Zhang RG, Adams PD. 2007. Downregulation 444 of Wnt signaling is a trigger for formation of facultative heterochromatin and onset of cell 445 senescence in primary human cells. Molecular Cell 27:183-196 DOI: $446 \quad 10.1016 /$ j.molcel.2007.05.034. 
447 Yen AHH, Sharpe PT. 2006. Regeneration of teeth using stem cell-based tissue engineering. 448 Expert Opinion on Biological Therapy 6:9-16 DOI: 10.1517/14712598.6.1.9.

449 Zhang DD, Park BM, Kang M, Nam H, Kim EJ, Bae C, Lim SK. 2016. The systemic effects of 450 sclerostin overexpression using Phi C31 integrase in mice. Biochemical and Biophysical 451 Research Communications 472:471-476 DOI: 10.1016/j.bbrc.2016.01.178.

452 Zhou H, Yang M, Li M, Cui L. 2017. Radial artery sclerostin expression in chronic kidney

453 disease stage 5 predialysis patients: a cross-sectional observational study. International Urology 454 and Nephrology 49:1433-1437 DOI: 10.1007/s11255-017-1604-0.

455 Zou J, Zhang W, Li XL. 2017. Effects of SOST gene silencing on proliferation, apoptosis, 456 invasion, and migration of human osteosarcoma cells through the Wnt/ $\beta$-catenin signaling 457 pathway. Calcified Tissue International 100:551-564 DOI: 10.1007/s00223-016-0231-6. 


\section{Figure 1}

Expressions of sclerostin in senescent dental pulp, subculture-induced senescent HDPCs and lentiviral infected HDPCs.

(A) Immunohistochemical staining for sclerostin in young dental pulps; (B)

immunohistochemical staining for sclerostin in senescent dental pulps (green arrows point to odontoblasts and red arrows point to dental pulp cells); d, dentin; pd, predentin; p, pulp; ob, odontoblast; scale bar $=20 \mu \mathrm{m}$. (C) qRT-PCR analyses of expression levels of sclerostin, p16, p53, and p21 in young and senescent dental pulps; (D) qRT-PCR analyses of expression levels of sclerostin, p16, p53, and p21 in subculture-induced senescent HDPCs. (E) qRT-PCR and (F) Western blot analyses of sclerostin expressions in HDPCs infected with control vector and SOST; $(\mathrm{G})$ qRT-PCR and $(\mathrm{H})$ Western blot analyses of sclerostin expression in HDPCs infected with sh-control vector and sh-SOST. (** $p<0.01$; *** $P<0.001$ )

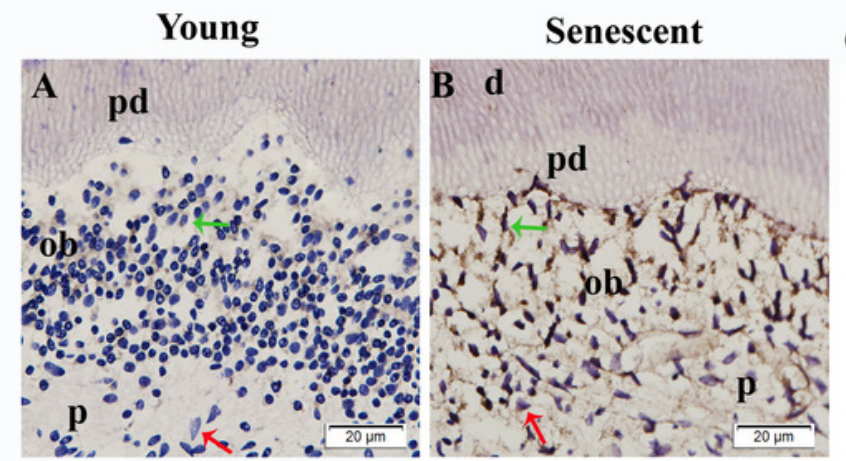

C

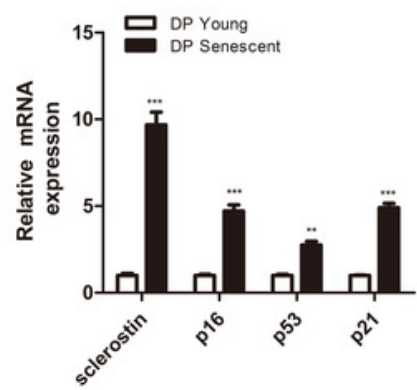

$\mathbf{E}$

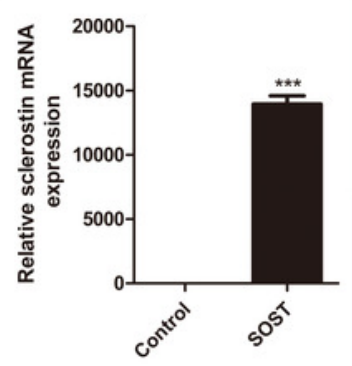

$\mathbf{F}$

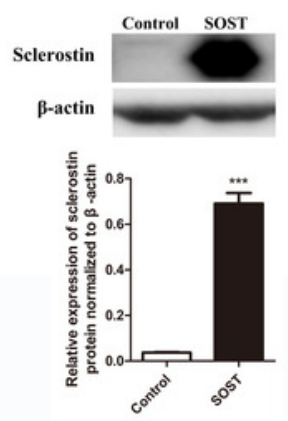

G

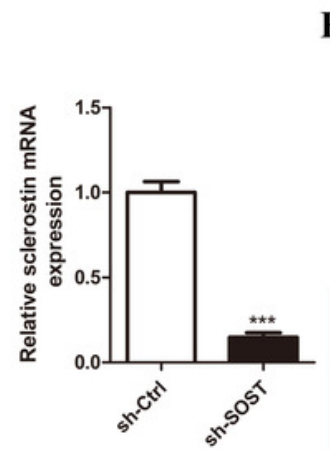

D

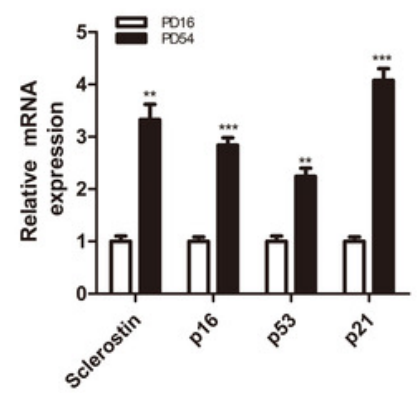

H

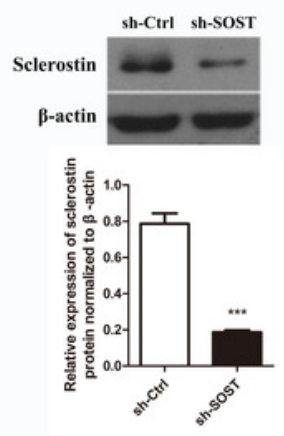




\section{Figure 2}

Effects of sclerostin overexpression and knockdown on senescence and proliferation of HDPCS.

(A) SA- $\beta$-Gal staining and (B) cell proliferation activity of Control and SOST HDPCS; (C) qRTPCR analyses of expression levels of p16, p53, and p21 in Control and SOST HDPCs. (D) SA- $\beta$ Gal staining and (E) cell proliferation activity of sh-Ctrl and sh-SOST HDPCs; (F) qRT-PCR analyses of expression levels of p16, p53, and p21 in sh-Ctrl and sh-SOST HDPCs. $(* p<0.05$, ** $p<0.01 ; * * * P<0.001)$
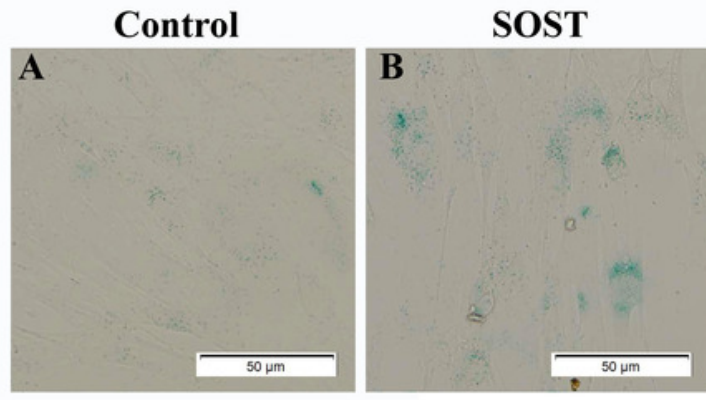

C

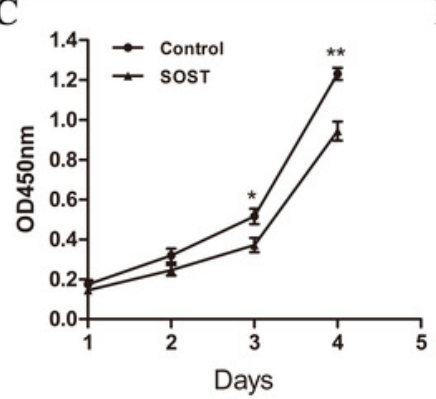

sh-Ctrl

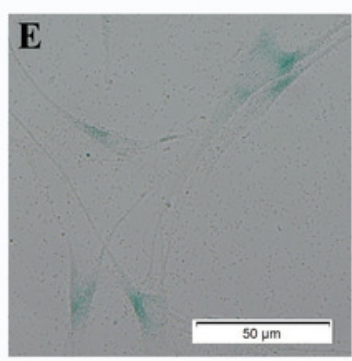

sh-SOST

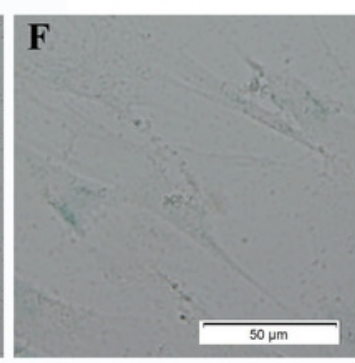

G

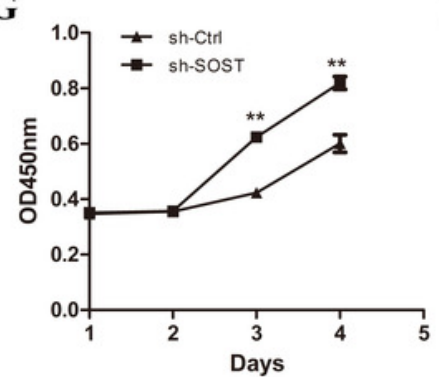

D

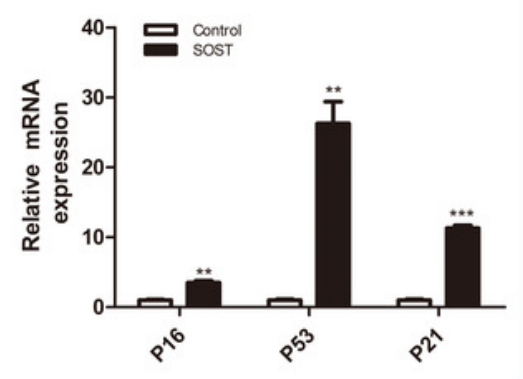

H

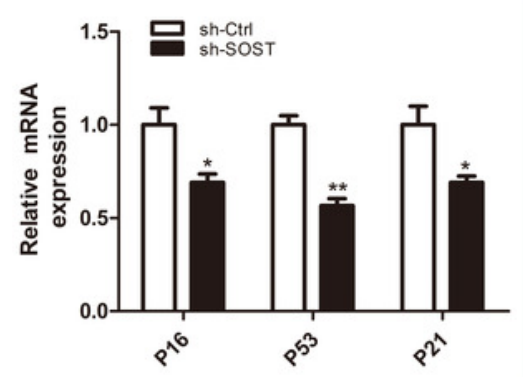




\section{Figure 3}

Effects of sclerostin on odontoblastic differentiation of HDPCs.

(A) ALP staining and ALP activity of early-passaged Control and SOST HDPCs on day 7; (B) alizarin red staining for mineral nodule formation of early-passaged Control and SOST HDPCS on day 14; (C) qRT-PCR analyses of expression levels of odontoblastic markers in earlypassaged Control and SOST HDPCs on day 7. (D) ALP staining and ALP activity of latepassaged sh-Ctrl and sh-SOST HDPCs on day 7; (E) alizarin red staining for mineral nodule formation of late-passaged sh-Ctrl and sh-SOST HDPCs on day 14; (F) qRT-PCR analyses of odontoblastic markers in late-passaged sh-Ctrl and sh-SOST HDPCs on day 7. $\left({ }^{*} p<0.05,{ }^{* *} p\right.$ $<0.01 ; * * * P<0.001)$ 
A
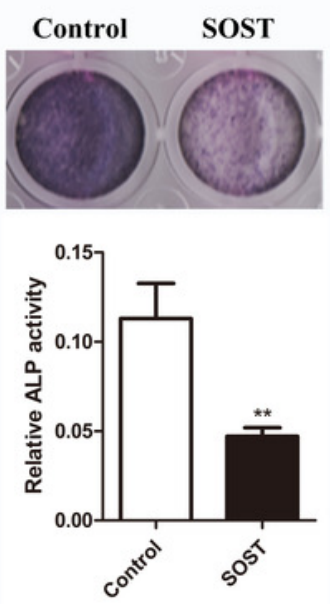

D
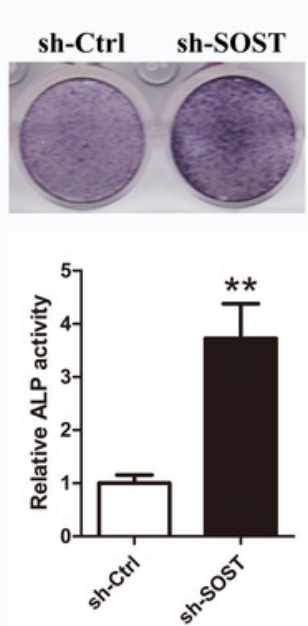

B
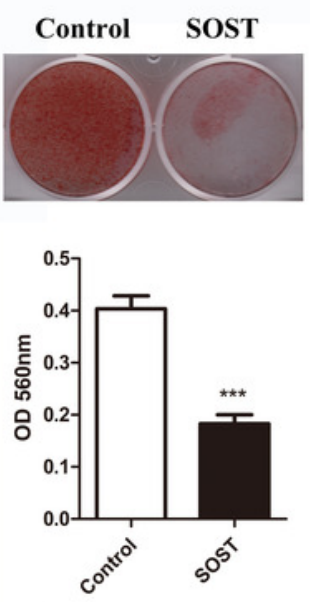

$\mathbf{E}$
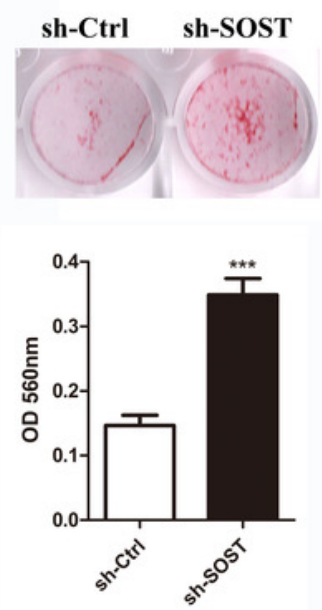

C

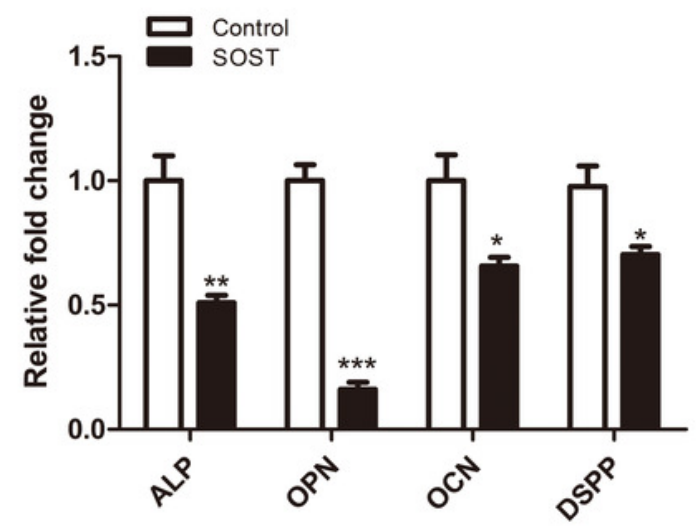

$\mathbf{F}$

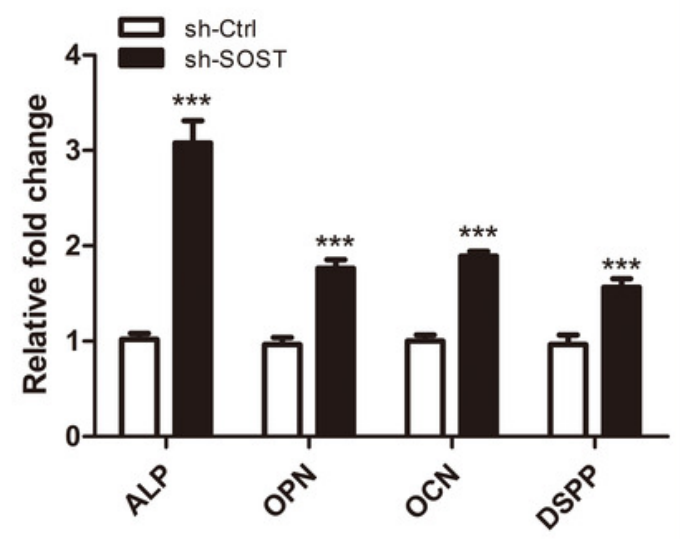


Figure 4

Effect of sclerostin on Wnt/ $\beta$-catenin pathway.

(A) Western blot analysis of $p$ - $\beta$-catenin expression in early-passaged Control and SOST HDPCs; (B) Western blot analysis of $\mathrm{p}-\beta$-catenin expression in late passaged sh-Ctrl and shSOST HDPCs. $(* p<0.05, * * p<0.01)$

A
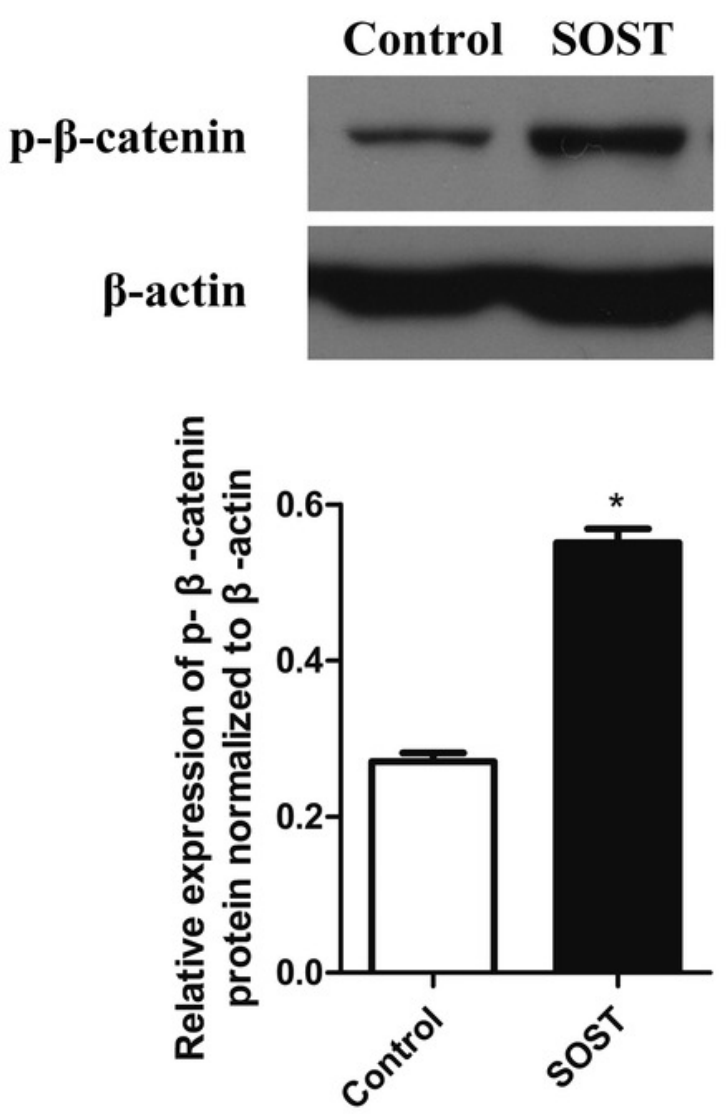

B
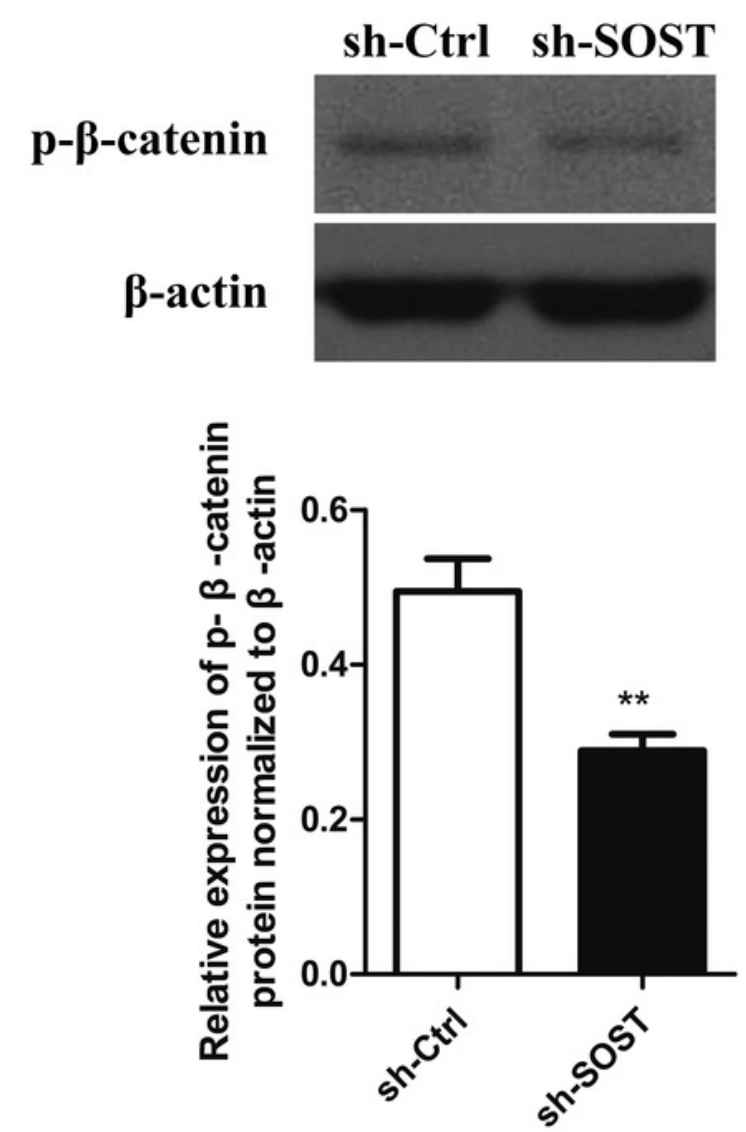
Table $\mathbf{1}$ (on next page)

Primer Sequences Used for Real-time PCR. 
1 Table 1 Primer Sequences Used for Real-time PCR

\begin{tabular}{lll}
\hline Genes & Forward(5'-3') & Reverse(5'-3') \\
\hline GAPDH & AACAGCGACACCCACTCCTC & CATACCAGGAAATGAGCTTGACAA \\
ALP & CGAGATACAAGCACTCCCACTTC & CTGTTCAGCTCGTACTGCATGTC \\
OPN & GCCGAGGTGATAGTGTGGTT & CAACGGGGATGGCCTTGTAT \\
OCN & GGTGCAGCCTTTGTGTCCAA & CCTGAAAGCCGATGTGGTCA \\
DSPP & CAACCATAGAGAAAGCAAACGCG & TTTCTGTTGCCACTGCTGGGAC \\
Sclerostin & TGGCAGGCGTTCAAGAATGA & GCCCGGTTCATGGTCTTGTT \\
P16 & CCCAACGCACCGAATAGTTAC & CAGCAGCTCCGCCACTC \\
P53 & ACCTATGGAAACTACTTCCTGAAA & CTGGCATTCTGGGAGCTTCA \\
P21 & TCAGGGTCGAAAACGGCG & CCTCTTGGAGAAGATCAGCCG
\end{tabular}

2 